\section{PSICOLOGIA IBEROAMERICANA}

\section{Psicología lberoamericana}

ISSN: 1405-0943

psicología.iberoamericana@uia.mx

Universidad Iberoamericana, Ciudad de

México

México

Méndez Lara, María del Rocío; Galvanovskis Kasparane, Agris

Sentido de Comunidad Virtual: Un Estudio Teórico Empírico

Psicología Iberoamericana, vol. 19, núm. 1, enero-junio, 2011, pp. 8-18

Universidad Iberoamericana, Ciudad de México

Distrito Federal, México

Disponible en: http://www.redalyc.org/articulo.oa?id=133920896002

Cómo citar el artículo

- Número completo

- Más información del artículo

Página de la revista en redalyc.org

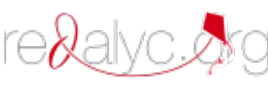

Sistema de Información Científica

Red de Revistas Científicas de América Latina, el Caribe, España y Portugal

Proyecto académico sin fines de lucro, desarrollado bajo la iniciativa de acceso abierto 


\title{
Sentido de Comunidad Virtual: Un Estudio Teórico Empírico
}

\author{
Sense of Virtual Community: A Theoretical and Empirical Study
}

\author{
María del Rocío Méndez Lara \\ Agris Galvanovskis Kasparane* \\ Universidad de las Américas, Puebla, México
}

\section{RESUMEN}

En los últimos años, las comunidades virtuales han tenido un fuerte desarrollo. La filosofía web 2.0 ha logrado contar con interfases más sofisticadas, enfocadas principalmente al intercambio de información, y la construcción y generación social del conocimiento; ejemplo de ello son las redes sociales y foros de discusión, entre otras. Para preservar este conocimiento es importante mantenerlas activas, y el sentido de comunidad virtual (SCV) juega un papel preponderante. Son pocas las investigaciones que se han realizado para identificar la estructura del SCV, y las existentes se han basado, casi todas, en el marco teórico de las comunidades físicas. Debido a esto, la presente investigación tiene como objetivo identificar y proponer los conceptos que miden SCV que nos permitan, a futuro, crear una escala para monitorear estas comunidades y lograr su permanencia y la preservación del conocimiento de las mismas.

Descriptores: Sentido de comunidad virtual, Internet, comunidades virtuales, redes sociales, sentido de comunidad, sentido de pertenencia, comunidades web 2.0.

\section{ABSTRACT}

In recent years, virtual communities have had a strong development. These communities are Web 2.0 based technologies and focused mainly on the exchange of information and the social construction of knowledge. An example of these technologies are social networks, forums, collaborative tagging systems and other applications that enable people to connect with friends, share information and knowledge, build relationships and collaborate with others. To preserve this knowledge, it is important to keep these communities active and in order to do so the sense of virtual community (SOVC) plays a leading role. Most researchers have adapted sense of community theories, to virtual groups. This work reviews the empirical studies on SOVC, examines and proposes a theoretical model and the dimensions of SOCV that allow us to develop a measure to monitor these communities and ensure their continuity and preservation of knowledge.

Key words: Communities, Internet, Computer mediated communication, Community involvement, Community involvement, Motivation, Online social networks

\footnotetext{
* Para correspondencia: María del Rocío Méndez Lara: Exhacienda Sta. Catarina Mártir, San Andrés. Cholula, Puebla. C.P. 72000. Tel. (222) 229-2124, rocio.mendez@udlap.mx. Agris Galvanovskis Kasparane: Exhacienda Sta. Catarina Mártir, San Andrés. Cholula, Puebla. C.P. 72000. Tel. (222) 2292486, agris.galvanovskis@udlap.mx.
} 


\section{INTRODUCCIÓN}

Internet, llamada comúnmente "red de redes," ha tenido un fuerte impacto en los diferentes ámbitos económico, educativo, social y tecnológico. En especial, ha traído consigo la oportunidad de una comunicación y relación de manera virtual entre una gran cantidad de personas en cualquier lugar. Las comunidades virtuales se definen como un grupo de personas que interactúan y se comunican a través de la computadora. Éstas, son grupos sociales que están en la red y que tienen una población suficientemente grande para discutir sobre un tema (Rheingold, 1996). Las estadísticas muestran que el $84 \%$ de los usuarios de Internet se han contactado o participado en una comunidad virtual (Porter, 2004). En la actualidad hay diferentes tipos, que van desde los listservs, foros de discusión como yahoo, redes sociales como Facebook, Ning, Hi5, Twitter, Wikis; como Wikipedia, sistemas de etiquetado colaborativo para compartir fotos como Flickr, videos como Youtube, documentos como Scribd, presentaciones como Slideshare, sitios web como Delicious, entre otras. La mayoría son parte de las tecnologías web 2.0, concepto acuñado por Dale Dougherty y Tim O'Reilly para referirse a herramientas enfocadas a compartir información, construir y generar conocimiento entre los miembros de la comunidad para su uso común (O’Reilly, 2005). Para lo cual cuentan con sistemas que les permiten interactuar, tener un historial sobre sus discusiones; compartir, organizar y clasificar información para su futuro acceso. En las comunidades virtuales se comparte actualmente información y conocimiento, además de un entendimiento mutuo. Para su buen funcionamiento es importante saber qué papel juega cada miembro de esa comunidad. Se desarrollan sentimientos de membresía, distribución de roles e identidades, y se establece un sentido de comunidad virtual (SCV). Cuando no existe este sentido de comunidad, por lo general éstas tienden a desaparecer o los miembros dejan de participar. Nielsen (2006) menciona que sólo un 1\% de los miembros en una comunidad participan, y un $90 \%$ sólo está inscrito pero no tiene ninguna participación. Debido a lo anterior es importante mantenerlas activas y lograr que los miembros participen, con el fin de preservar este conocimiento y para lograrlo, el SCV es vital. Si no existiera el sentido de comunidad, éstas desaparecerían y como consecuencia el conocimiento no se preservaría.
Entre los principales factores del sentido de comunidad virtual se encuentran: membresía, límites, pertenencia, símbolos grupales, normas de esfuerzo y reto, apoyo entre miembros, conexión emocional entre los miembros, entre otros.

Blanchard (2007) fue uno de los pocos investigadores que estudió este concepto con más detalle. Desarrolló un instrumento para medirlo, adaptando los reactivos del Índice de Sentido de Comunidad (McMillan \& Chavis, 1986) aplicado a comunidades físicas. Utilizó una muestra de 265 sujetos con tres listservs y 4 foros (Usenet newsgroups). Concluyó que existen problemas de validez en dicho índice. Esta investigación se basó principalmente en listservs y foros, pero en la actualidad, con la filosofía web 2.0, los sistemas para la creación de comunidades virtuales han crecido de manera exponencial.

Algunos estudios (Doolittle \& MacDonald, 1978; Glynn, 1981, citado por Kruger, et al., 2001; Obst, 2004; Blanchard \& Markus, 2004; Blanchard, 2007; Moore \& Serva, 2007; Yu, Jiang \& Chuan, 2007) han mostrado que existen más y diferentes factores que podrían medir el sentido de comunidad virtual y que no han sido incluídos en las anteriores escalas. Debido a esto, el objetivo de este estudio es identificar y analizar los conceptos que podrían constituir SCV, aplicado principalmente a estos nuevos sistemas web 2.0 y después utilizarlos para crear una nueva escala que permita monitorear estas comunidades para lograr su permanencia. De esta manera, la información y el conocimiento compartido no se perderían.

\section{COMUNIDADES VIRTUALES (CV)}

Webster (2010) define "comunidad" como un conjunto de personas viviendo con intereses comúnes en un área particular o "un conjunto de personas con intereses profesionales comunes. El concepto "comunidad virtual" se usa para describir diferentes formas de comunicación mediada por computadora, el cuál es usado por un grupo de personas (Cheon \& Ahn, 2009). Wellman (citado por Obst, 2004) afirma que la "comunidad" está constituida por las redes de lazos interpersonales que proveen sociabilidad, apoyo social, información, sentido de pertenencia e identidad social. 
Chavis y Newbrough (1986) mencionan que se trata de cualquier conjunto de relaciones sociales enlazadas por un sentido de comunidad.

En las CV, las personas forman una red social donde comparten información y conocimiento socializando y haciendo diferentes transacciones. Utilizan un lenguaje común, una comunicación simple, espacio público, intereses comunes, valores, metas, la persistencia de un significado común; usan tecnologías de la información para la interacción, sin barreras de espacio y tiempo e identidades digitalizadas como substitutos de seres físicos. Room (citado por Wang \& Chen, 2004) argumenta que las comunidades virtuales redefinirán las relaciones interpersonales.

Se considera que Rheingold es uno de los investigadores que ha trabajado más en el campo de las comunidades virtuales (Rheingold, 1996 \& Tilley, 2006). Rheingold (1996) es el primero que contribuye a la difusión del término "comunidad virtual" (CV), subrayando el lado humano en ellas. Definió comunidades virtuales como agrupaciones culturales que emergen cuando una gran cantidad de personas se encuentra en el ciberespacio. Posteriormente, afinó su definición y las describió como un grupo de gente que podría o no conocer a otros cara a cara para intercambiar palabras e ideas a través de la mediación de la computadora y las redes. Considera que la comunidad virtual emerge de la red con sentimientos y relaciones personales en el ciberespacio. Este autor fue un determinista tecnológico porque concluyó que existe una relación previsible entre la tecnología y el comportamiento de la gente. Está convencido de que las comunicaciones mediadas por computadora (CMC) tienen el potencial de cambiar vidas de los participantes en varios aspectos: a) el comportamiento humano individual; b) la interacción persona-persona y c) el comportamiento político.

En el ciberespacio (Rheingold, 1996) se tiene la oportunidad de chatear, argumentar, realizar actividades de comercio, intercambiar conocimiento, compartir emociones, hacer planes, enamorarse, chismear, discutir, encontrar amigos, perderlos, jugar, etcétera. Las identidades de los participantes convergen e interactuan electrónicamente sin tomar en cuenta el tiempo o el lugar. El ciberespacio (Lyman, 1999) puede evocar un sentido de lugar y de comunidad intelectual, por lo que el término "comunidad virtual" incluye sentimien- tos de solidaridad social manifestados por medio de un software interactivo de red. Una comunidad en línea consta de personas que interactúan socialmente y se esfuerzan para satisfacer sus propias necesidades o tienen sus roles especiales; un propósito compartido que provee una razón para la comunidad, políticas que guían las interacciones de las personas, y sistemas de computación para apoyar y mediar la interacción social, además de facilitar un sentido de fraternidad (Preece, citado por Garber, 2004).

Las comunidades virtuales han tenido un desarrollo permanente, sobre todo en Estados Unidos. Usenet fue una de las primeras comunidades. El chat fue uno de los sistemas más difundidos. Cerca de 1996 nacen nuevas herramientas para la participación en comunidades virtuales, en específico las basadas en la filosofía web 2.0 como las redes sociales, sistemas de contenido, Wikis, blogs, entre otras. Éstas permiten a las personas comunicarse con amigos, compartir actividades e intereses. Algunas de ellas son Facebook, MySpace, Delicious, Flickr, Scribd, Wikilearning, entre otras. Aquí las personas comparten ideas e intereses, crean relaciones sociales con otros, etc. Cheon y Ahn (2009), proponen categorías para agrupar estas nuevas herramientas: a) foros, que son discusiones sobre un tema; b) bases de conocimiento, proveen y permiten compartir información digitalizada, c) colaboración abierta, ambientes para crear y modificar contenidos; d) páginas personales, como los blogs, los cuales son mantenidos y creados por un solo individuo y e) los mundos virtuales, que son ambientes simulados por computadora donde un avatar es la identidad del usuario. Estas comunidades han aumentado considerablemente su número de usuarios. Un ejemplo es Facebook, que actualmente tiene 400 millones en todo el mundo. Los usuarios de estas comunidades son principalmente adolescentes, estudiantes universitarios y jóvenes de 20 años en adelante. Todos estos nuevos sistemas han llevado a que las personas compartan, colaboren e enriquezcan en especial información y conocimiento. El enfoque de todos estos sistemas es dar y obtener información, pericia, opinión a través de un sentido de reciprocidad y obligación moral. La sabiduría parece ser un factor único motivacional para estos sistemas (Brunold, Merz \& Wagner, 2002). Como se ha visto por muchos años, estas comunidades aparecen y de- 
saparecen. En México, el 64\% de los usuarios de los diferentes sitios de Internet son menores de 25 años y llegan a invertir más de medio día al entretenimiento como el uso de redes sociales y otro tipo de comunidades (Morales, 2010).

\section{SENTIDO DE COMUNIDAD VIRTUAL (SCV)}

El sentido de comunidad (SC) cara a cara, en general, es el sentimiento de conexión y pertenencia a un grupo social, y conduce a importantes resultados en comunidades humanas y organizaciones cara a cara (Blanchard \& Markus, 2002).

El trabajo de Sarason (1977, citado por Obst \& White, 2004) fue pionero en la psicología del sentido de comunidad. Posteriormente McMillan y Chavis (1986) propusieron un modelo con cuatro componentes: a) membresía, constituido por una serie de factores que interactuán en la comunidad (quién está dentro o fuera): una historia compartida, símbolos comunes, seguridad emocional e inversión personal; la membresía permite sentirse como parte de la comunidad con derechos y responsabilidades; b) influencia, es un proceso interno que refleja la importancia que una persona tiene a través de las decisiones y acciones de la comunidad, permite al individuo hacer su contribución y cierto nivel de libertad de expresión; c) integración y realización de necesidades, es la motivación que los miembros de la comunidad están recibiendo al cubrir sus necesidades como status, demostración de competencia en algún área, valores compartidos, etcétera, lo cual refuerza la membresía de la comunidad y d) conexión emocional compartida, enfocada a compartir en la comunidad eventos significativos y nivel de contacto entre ellos.

Uno de los instrumentos más usados para medir el sentido de comunidad virtual es el índice de sentido de comunidad (ISC), desarrollado por McMillan y Chavis (1986), quienes utilizan los cuatro factores anteriormente mencionados. Se ha asociado con relaciones predictivas, a través de diferentes tipos de comunidades, edades, grupos y culturas. Ha sido una medida útil, validada y confiable. Sin embargo, las propiedades psicométricas inconsistentes de las sub-escalas han hecho que los investigadores tengan que eliminar reactivos para mejorar éstas. Los análisis factoriales no han mostrado evidencia empírica para apoyar la visión de los autores como un constructo multidimensional (Fisher, Sonn \& Bishop, 2002, citado por Blanchard, 2007).

Otras medidas han sido desarrolladas combinando el ISC con otros reactivos e instrumentos (Allen \& Allen, 1987; Berger, 1987, Davison \& Cotter, 1993; Glynn, 1981; Royal \& Rossy, 1996). McMillan (citado por Blanchard \& Markus, 2004) ha reconceptualizado el término incluyendo aspectos espírituales y artísticos de las comunidades.

Respecto al sentido de comunidad virtual, Blanchard (2007) lo define como sentimientos de membresía, identidad, afiliación y vinculación al grupo que interactúa principalmente a través de una comunicación electrónica dentro de una comunidad virtual. Son pocos los estudios que se han realizado sobre SCV. Se ha encontrado que la seguridad percibida, seguridad verbal y respeto entre los miembros son factores relacionados se relacionan con éste (Doolittle \& MacDonald, 1978; Glynn, 198, citado por Kruger, et al., 2001). Otro concepto es la conexión emocional compartida entre los participantes (Greer, 2000; Preece, 1999; Rheingold, 1993, citado por Blanchard \& Markus, 2004). Existe evidencia de que una comunidad vrtual puede proveer apoyo social significativo. Hay influencia en términos de esfuerzo y normas con reto (Baym, 1997; Kollock \& Smith, 1994; Markus, 1994, McLaughlin, et al., 1995; Pliskin \& Romm, 1997, citados por Blanckard \& Markus, 2004). Varios estudios (Baym, 1997; Greer, 2000; Preece, 1999; citados por Blanckard \& Markus, 2004) y Rheingold (1993) indican que el intercambio y apoyo entre los miembros son los sentimientos más importantes en las comunidades virtuales.

Cheon y Ahn (2009) propusieron seis categorías que motivan a los miembros de una comunidad virtual a participar como a) valor hedónico, relacionado con el placer de participar; b) altruismo, sentir la obligación de ayudar; c) entendimiento, compartir su conocimiento; d) social, socializar, tener conexiones emocionales y hacer amigos; e) carrera, aumentar el conocimiento propio, aumentar el ego por la participación y mejorar la autoestima, entre otras.

En las comunidades virtuales existe membresía, límites, pertenencia y símbolos grupales (Baym, 1995, 1997; Curtis, 1997; Greer, 2000; Herring, 1996; Kollock \& Smith, 1994; Markus, et al., 2000, Phillips, 1996, citados en Blanchard y Markus, 2004). 
Bingshan (2010) menciona que los factores que influyen en el sentido de comunidad virtual son: las características personales como género, carrera, salario y comportamiento en línea, la conexión de manera física, la administración de la comunidad como: opinión del líder, contenidos, reglas, y finalmente los comportamientos en la comunidad y los factores tecnológicos como: plataforma, diseño del sitio y navegación.

Koh y Kim (2003) encontraron que el entretenimiento es importante para obtener una evaluación positiva, así como afecto de la comunidad virtual; dicha evaluación se refiere al placer o alegría derivada de las interacciones con los miembros y contenido que hay en la comunidad virtual. La emoción, placer y satisfacción resultan de una experiencia alegre. El entretenimiento puede ayudar a percibir afiliación a la comunidad porque los miembros necesitan conocerse por medio de las interacciones entre ellos o con el contenido para que en la comunidad se desarrolle la lealtad. Con este factor, la inmersión y la membresía incrementan. Koh y Kim (2003) distinguieron tres dimensiones en el sentido de comunidad virtual: a) membresía, es el sentimiento de pertenencia, b) la influencia, permite hacer una diferencia en su comunidad virtual y c) la inmersión, refleja el grado en el cual el comportamiento de los miembros de la comunidad está dominado por el estado de flujo causado por la interacción humano-computadora. Posteriormente en otro estudio, Koh y Kim (2007) describieron otras características de las comunidades virtuales: a) entusiasmo del líder, que se refiere a ayudar a los miembros para que sientan que la comunidad virtual está activa, que hay atención y cuidado por promoverla; b) actividades fuera de línea, el nivel de la presencia social en otros ambientes puede influir en el sentido de comunidad virtual; c) el valor o la utilidad de lo que se comparte y las d) facilidades físicas, como una adecuada infraestructura de tecnologías de información para contar con una fácil interacción e intefases amigables. En un newsgroup llamado MSN, Blanchard y Markus (2004) encontraron que para tener sentido de comunidad virtual los principales aspectos son los siguientes: a) reconocimiento, el interés en reconocer el trabajo de los demás y que además sean reconocidos ellos mismos; b) identificación, que es la identidad del participante en la comunidad virtual desarrollada por medio de los mensajes que envían; se puede identificar en los comentarios recibidos de cada miembro; c) apoyo, o sea, dar y recibir apoyo informacional y socio-emocional, porque los miembros consideran que la comunidad es útil para conocer las necesidades de los demás; d) relación con otros miembros, se refiere al desarrollo de amistad entre los miembros a través de una comunicación privada en línea; e) vínculos emocionales, se puede experimentar niveles diferentes de vínculos con la comunidad; y f) obligación, compromiso con la comunidad virtual. Encontraron que los que más participan sienten mayor obligación con la comunidad. Entre los resultados que obtuvieron, destaca que los miembros del grupo virtual no reportaron el sentimiento de influencia o bien, fueron influenciados por otros. El reconocimiento y las relaciones fueron importantes para el sentido de comunidad virtual. Al igual que Obst y colegas (2002) encontraron sentimientos de membresía. Esto incluye sentimientos individuales de identidad con los miembros de la comunidad. Blanchard y Markus (2004) proponen que las diferencias en influencia y la importancia de reconocer y tener relaciones con individuos podrían ser los más relevantes para interactuar en ambientes virtuales. Joinson y Diez Uhler (citados por Blanchard, 2008) mencionaron que usando las tecnologías de información y comunicación (TIC), las interacciones son generalmente anónimas y los miembros podrían tener preocupación acerca de las verdaderas identidades de los otros. Determinaron que la identidad y la identificación juegan un rol importante en el comportamiento informacional y afectivo en línea para los que reciben la información, pero para el que quiere compartir, el anonimato le permite tener una mayor inmersión en el grupo e incrementar la identidad en la comunidad teniendo también varios resultados positivos tales como solidaridad y unidad. La presencia de información que identifique al individuo: nombre o foto contribuye a construir la identidad individual dentro de un grupo en línea. Cuando la identidad de algún participante es sobresaliente, otros miembros sobre-interpretan e idealizan los patrones de su coducta (Walther, 1996 citado por Blanchard, 2008).

Postmes, Spears, Lee y Novak (2005 citados por Blanchard, 2008) sugirieron que la identidad individual y de grupo pueden coexistir y que las expresiones 
de individualidad a través de la comunicación de los miembros podrían fortalecer la identidad y solidaridad del grupo.

Blanchard (2008) aplicó la teoría del intercambio social al estudio de las comunidades virtuales, subra yando que el apoyo recibido y proporcionado es muy importante en una comunidad virtual. Desde este punto de vista, el apoyo recibido y proporcionado debe ser recíproco y equivalente entre sí.

Compranzano \& Mitchell (2005; citados por Blanchard, 2008) comentaron que la gente se siente obligada a ciertas reglas de intercambio, a normas que sirven como guías para las interacciones con otros. Estas normas de comportamiento determinan cómo la gente participa en el intercambio, así mismo, intervienen en la definición de los límites del grupo.

Para la construcción del instrumento de medición de sentido de comunidad virtual, se ha usado como base el instrumento "Índice de Sentido de Comunidad (ISC), creado por McMillan y Chavis (1986). Blanchard (2007) encontró que éste no posee suficiente validez de contenido para las comunidades virtuales. De hecho, varios ítems no son relevantes. Para adaptar ISC a las comunidades virtuales Blanchard (2007) tomó 22 reactivos de ISC, los cuales fueron modificados: cambiando cuadra por grupo y vecinos por miembros. Se crearon 10 nuevos reactivos para evaluar los componentes únicos de SCV, mismos que estuvieron relacionados con la identificación y el intercambio de información como apoyo. Este cuestionario fue elaborado principalmente con un enfoque de comunidades virtuales web 1.0 y tomando como base las dimensiones de McMillan y Chavis (1986), las cuales fueron creadas para una comunidad física (cara a cara).

Con lo anterior se puede resumir, primero, que la mayoría de la información que se ha rescatado de las diferentes investigaciones sobre SCV ha sido descriptiva, por lo que las interpretaciones son diversas, y segundo, los modelos de SCV están limitados porque la mayoría se ha basado en la conceptualización de McMillan y Chavis (1986) en comunidades físicas cara a cara (Ellonen, Kosonen \& Henttonen, 2007). Debido a lo anterior, se analizarán e identificarán empíricamente las categorías que se relacionan con sentido de comunidades virtuales actuales.
Para fines de esta investigación, comunidades virtuales se definió como "conglomerados sociales que emergen de la red cuando las personas tienen una diversidad de discusiones públicas con suficientes sentimientos humanos para formar redes de relaciones personales en el ciberespacio (Chin \& Chignell, 2006; Reinghold, 2010), y sentido de comunidad virtual se define como el reconocimiento, identificación, relación, membresía, influencia, integración y realización de necesidades, conexión compartida, apoyo y funcionamiento de la interfaz dentro de una comunidad virtual.

\section{MÉTODO}

\section{Participantes}

Para identificar los conceptos principales relacionados con SCV se aplicó una encuesta a 150 estudiantes universitarios, de los cuales 122 la contestaron completamente. Las 28 excluidas fueron repondidas errónea o parcialmente. El requisito para que contestaran la encuesta fue que pertenecieran a diferentes comunidades virtuales, como foros, redes sociales, sistema de etiquetado colaborativo, etcétera, y que sus edades fluctuaran entre 18 y 25 años. Se estableció este rango de edad ya que la literatura muestra que los usuarios potenciales de estas comunidades son menores de 25 años (Morales, 2010). Los grupos fueron seleccionados al azar en cinco diferentes instituciones universitarias.

\section{Instrumento y procedimiento}

Para realizar esta investigación se decidió primero realizar una revisión de la literatura con el objetivo de identificar los conceptos que podrían estar relacionados con SCV. Al hacerlo, se identificaron 28 factores relacionados directa o indirectamente con sentido de comunidad virtual. Después se procedió a aplicar una encuesta anónima para identificar de modo empírico los conceptos que miden SCV. Este instrumento contó con dos secciones: en la primera, se incluyó la definición de sentido de comunidad y se les pidió a los encuestados que marcaran de un listado de 28 conceptos definidos cuáles consideraban que podrían influir en el SCV. En la segunda sección, se les hizo una pregunta abierta para que mencionaran cuáles eran los conceptos relacionados con esta variable. A los estudiantes se 
les invitó a contestar la encuesta y se les explicó cómo deberían responderla. Con los resultados de esta encuesta fue posible identificar los principales conceptos relacionados con sentido de comunidad virtual, los cuáles podrán ser la base para crear a futuro un instrumento que mida mejor SCV.

\section{RESULTADOS}

Después de realizar una revisión de la literatura sobre los diferentes factores que integran el concepto de sentido de comunidad virtual se identificaron un total 28 factores (Baym, 1995, 1997; Blanchard, 2007; Curtis, 1997; Doolittle \& MacDonald, 1978; Dunham, et al., 1998; Glynn, 1981; Greer, 2000; Herring, 1996; Kollock
\& Smith, 1994; Markus, et al., 1994, 2000; McLaughlin, et al., 1995; Moore \& Serva, 2007; Phillips, 1996; Pliskin \& Romm, 1997; Preece, 1999; Rheingold, 1993; Yu, Jiang \& Chuan, 2007), mismos que se presentan en la Tabla 1.

Tomando en cuenta estos factores se aplicó la encuesta, la cual tuvo como objetivos: 1) seleccionar de los 28 conceptos aquellos que consideraran lo más relacionados con SCV y 2) por medio de una pregunta abierta, conocer qué aspectos consideraban más importantes para tener SCV.

Con los resultados de esta aplicación y un proceso de categorización se lograron identificar 9 conceptos. La Tabla 2 los muestra.

Tabla 1. Factores identificados en la literatura sobre sentido de comunidad virtual

\begin{tabular}{|l|l|l|l|}
\hline 1. Valores & 8. Reputación & 15. Vínculos emocionales & 22. Diseño \\
\hline 2. Social & 9. Conocimiento & 16. Relación & 23. Interacción \\
\hline 3. Entendimiento & 10. Empatía & 17. Identificación & 24. Eventos diferentes \\
\hline 4. Carrera & 11. Emoción & 18. Reconocimiento & 25. Efecto de honor \\
\hline 5. Mejora y protección & 12. Afiliación & 19. Seguridad & $\begin{array}{c}\text { 26. Competencias en el } \\
\text { funcionamiento del grupo }\end{array}$ \\
\hline 6. Apoyo & 13. Confianza & 20. Cohesión & 27. Influencia \\
\hline 7. Autoexpresión & 14. Obligación & 21. Benevolencia & 28. Membresía \\
\hline
\end{tabular}

Tabla 2. Conceptos sobre SCV identificados por medio de una encuesta a una muestra de estudiantes universitarios

\begin{tabular}{|l|l|l|}
\hline Dimensiones de SCV & \multicolumn{1}{|c|}{ Definición } & \multicolumn{1}{c|}{ Autores } \\
\hline 1. Reconocimiento & $\begin{array}{l}\text { Es el nivel de reconocimiento sobre la información y el } \\
\text { conocimiento que comparte cada miembro por parte del } \\
\text { grupo y de la empresa y/o personas que crearon y administran } \\
\text { la comunidad }\end{array}$ & Blanchard y Markus (2004). \\
\hline 2. Identificación & $\begin{array}{l}\text { Las personas se identifican con el grupo por medio de los } \\
\text { comentarios y la información que comparten. Desarrollan } \\
\text { un entendimiento de las identidades de cada uno de los } \\
\text { miembros. Debe haber un entendimiento de la importancia de } \\
\text { compartir la información con todos y la voluntad para la ayuda } \\
\text { mutua, así como un sentimiento de obligación a participar } \\
\text { compartiendo información }\end{array}$ & $\begin{array}{l}\text { Blanchard y Markus, } \\
\text { (2004), Moore y Serva } \\
\text { (2007). }\end{array}$ \\
\hline
\end{tabular}




\begin{tabular}{|c|c|c|}
\hline Dimensiones de SCV & Definición & Autores \\
\hline 3. Relación & $\begin{array}{l}\text { Vínculos entre los miembros a través de una comunicación } \\
\text { en línea que permite cooperación, buena relación, interacción } \\
\text { constante, conocer a las personas, etc. La confianza entre los } \\
\text { participantes, lleva a una buena disposición para ayudarse. En } \\
\text { una buena relación debe existir credibilidad en la información } \\
\text { que comparte la gente; benevolencia, es decir que los } \\
\text { miembros del grupo realmente estén motivados e interesados } \\
\text { en ayudar a las personas; seguridad verbal, respeto entre los } \\
\text { miembros y expresión de emociones y opiniones }\end{array}$ & $\begin{array}{l}\text { Blanchard (2007); Blanchard } \\
\text { y Markus (2004); Doolitle y } \\
\text { MacDonald (1978); Glynn, } \\
\text { 1981; MacMillan y Chavis } \\
\text { (1986); Moore y Serva } \\
\text { (2007); Rovai (2002); Smith } \\
\text { (1975). }\end{array}$ \\
\hline 4. Membresía & $\begin{array}{l}\text { Sentimiento de saber que uno ha invertido tiempo en la } \\
\text { comunidad para llegar a ser parte y miembro, además de tener } \\
\text { derecho a pertenecer a ella. Involucra el sentimiento, creencia } \\
\text { y expectativas de que alguien es parte del grupo y que tienen } \\
\text { un lugar ahí, una sensación de aceptación por el grupo y } \\
\text { buena disposición para sacrificarse por él. Los símbolos son } \\
\text { parte de la membresía como logos, frases, cualquier evento } \\
\text { que sea identificado por el exterior }\end{array}$ & $\begin{array}{l}\text { Blanchard (2007); McMillan y } \\
\text { Chavis, (1986). }\end{array}$ \\
\hline 5. Influencia & $\begin{array}{l}\text { Los miembros necesitan sentir que ellos tienen influencia en el } \\
\text { grupo, sus ideas son aceptadas y hay un buen nivel de libertad } \\
\text { de expresión. Esta influencia permite cohesión }\end{array}$ & $\begin{array}{l}\text { Blanchard (2007); McMillan y } \\
\text { Chavis, (1986). }\end{array}$ \\
\hline $\begin{array}{l}\text { 6. Integración } \\
\text { y realización de } \\
\text { necesidades }\end{array}$ & $\begin{array}{l}\text { Beneficios que reciben las personas al ser miembros de la } \\
\text { comunidad. Es una retribución por ser miembro del grupo. } \\
\text { Las personas se atraen unas a otras cuando sus habilidades y } \\
\text { competencias pueden beneficiarlas, creándose un status al ser } \\
\text { miembro }\end{array}$ & $\begin{array}{l}\text { Berkowitz (1956); Blanchard } \\
\text { (2007); Hester, Roback, } \\
\text { Weitz, Anchor y McKee } \\
\text { (1976); Kelley (1951); } \\
\text { MacMillan y Chavis (1986); } \\
\text { Peterson y Martens (1972); } \\
\text { Sacks (1952); Zander y } \\
\text { Havelin (1960); Zander y } \\
\text { Cohen (1955). }\end{array}$ \\
\hline $\begin{array}{l}\text { 7. Conexión emocional } \\
\text { compartida }\end{array}$ & $\begin{array}{l}\text { Basada en una historia compartida. La interacción de } \\
\text { los miembros en eventos que comparten y los atributos } \\
\text { específicos de los eventos podrían facilitar o inhibir la fortaleza } \\
\text { de la comunidad.Entre más interactúe la gente más cohesión } \\
\text { existirá. Si la interacción es ambigua y las tareas de la } \\
\text { comunidad no se resuelven, disminuirá la cohesión del grupo }\end{array}$ & $\begin{array}{l}\text { Allan y Allan (1971); Cook } \\
\text { (1970); Festinger (1950); } \\
\text { Hamblin (1958); Sherif, } \\
\text { White y Harvey (1955); } \\
\text { Mann y Mann (1959); Wilson } \\
\text { y Miller (1961). }\end{array}$ \\
\hline $\begin{array}{l}\text { 8. Apoyo emocional } \\
\text { entre los miembros }\end{array}$ & $\begin{array}{l}\text { Ayuda mutua altruista entre los miembros para tener un } \\
\text { beneficio individual y grupal. Dar y recibir apoyo informacional } \\
\text { de calidad es muy importante porque se valora la información } \\
\text { compartida y la relación dentro de la comunidad, sintiendo } \\
\text { que se tiene la obligación moral de ayudar }\end{array}$ & $\begin{array}{l}\text { Blanchard y Markus, (2004); } \\
\text { Yu, Jiang y Chuan (2007). }\end{array}$ \\
\hline $\begin{array}{l}\text { 9. Funcionamiento de la } \\
\text { interfaz }\end{array}$ & $\begin{array}{l}\text { Capacidad de una interfaz de ser comprendida, aprendida, } \\
\text { usada y atractiva para el usuario para facilitar una interacción } \\
\text { efectiva. Relacionada con la capacidad de observación del } \\
\text { usuario, de recuperación de información, de sentimientos } \\
\text { relacionados con lo atractivo del sitio. Incluye letra, tamaño, } \\
\text { colores, simplicidad, flexibilidad, etc. }\end{array}$ & $\begin{array}{l}\text { Koh, Kim, Butler y Bock } \\
\text { (2007). }\end{array}$ \\
\hline
\end{tabular}

Los conceptos que se proponen son: 1) reconocimiento: que se refiere a las aportaciones de las personas. Esperan reconocimiento por parte del grupo y/o de los creadores de la comunidad. Los encuestados co- mentaron que las empresas que crean las interfases deberían desarrollar un programa de premiación para las mejores aportaciones, además; mencionaron la importancia que tiene para ellos sentirse cómodos al expre- 
sarse en la comunidad; 2) identificación: el 70\% mencionó la importancia de este aspecto. Consideraron necesario contar con un registro de integrantes para conocer mejor su entorno; 3) relación: el 85\% contestó que es necesaria una buena relación entre los miembros. El respeto y la honestidad son dos puntos imprescindibles; 4) membresía: en este punto los sujetos mencionaron que les interesa que el grupo los acepte y saber quiénes participan. Un $80 \%$ dijo que les gustaría saber qué piensan los demás miembros del grupo de ellos; 5) influencia: un $68 \%$ comentó que si sus comentarios influyeran en el pensamiento de los otros se sentirían más parte del grupo. Un $45 \%$ expresó la importancia de sentir que todos los participantes desean lo mismo de esa comunidad; 6) integración y realización de las necesidades: consideran que es necesario que exista un beneficio individual y grupal al participar, por lo que es importante saber los objetivos de la comunidad para conocer de antemano los beneficios de su participación; 7) conexión emocional compartida: entre más participaciones acertadas existen, hay una mayor cohesión. Esto puede desarrollar lazos afectivos y los miembros disfrutarán más al aportar; 8) apoyo emocional entre los miembros: en este punto, la calidad de la información compartida influye en el sentido de comunidad, y finalmente 9) funcionamiento de la interfaz: más de un $80 \%$ comentaron la importancia de este punto. Los encuestados mencionaron que la interfaz debe ser sencilla, atractiva, flexible, fácil de manejar y actualizada. Los puntos más mencionados se centraron en que las interfaces fueran fáciles y amigables. Este último resultado concuerda con los resultados de Koh, Kim, Butler y Bock (2007) y Bingshan (2010), quienes mostraron que las interfaces amigables y la confiabilidad del sistema afectan el acceso y uso del sitio web de la comunidad.

\section{DISCUSIÓN}

El objetivo de esta investigación fue recuperar y analizar los estudios que se han realizado sobre sentido de comunidad virtual, principalmente en comunidades virtuales basadas en la tecnologías web 2.0, e identificar los conceptos que están relacionados de manera directa con este factor. Después de ello, por medio de la aplicación de una encuesta se pudieron discriminar algunos, categorizarlos y seleccionar los más significa- tivos. De esta manera se está contribuyendo al desarrollo conceptual de SCV. Consideramos que este estudio, por medio de una exhaustiva revisión de la literatura y el punto de vista de los encuestados, encontró las evidencias necesarias para entender mejor cuáles son los factores relacionados con este concepto, lo cuál permitirá a futuro crear una escala que se adapte mejor a las actuales comunidades virtuales. Se sugiere que después los investigadores en el área puedan analizar los factores y proponer adiciones o exclusiones.

Como se puede ver, los cuatro conceptos aportados por McMillan y Chavis (1986), como membresía, influencia, integración y realización de necesidades y conexión emocional compartida se confirman en este estudio, así como en la investigación de Blanchard (2007). Aparecen otros conceptos, como el "reconocimiento" relacionado con la información que comparten, el cual concuerda con un trabajo que realizaron Blanchard y Markus (2004) donde este concepto se enfoca principalmente al reconocimiento por los miembros del grupo. En nuestra investigación los encuestados mencionaron la importancia del reconocimiento por parte de la empresa que creó el sistema. Respecto a los conceptos "identificación" y "relación," se confirman con la investigación de Blanchard y Markus (2004) y Moore y Serva (2007). Algo adicional que se pudo identificar es que para que exista una buena relación los encuestados consideran importantes la confianza entre los participantes, la credibilidad en la información que comparten, la seguridad verbal, enfocada principalmente al respeto en su expresión de emociones y opiniones. El "apoyo emocional entre los miembros", se confirma con las investigaciones de Blanchard y Markus (2007) y Yu, Jiang y Chuan (2007). Algo interesante que se identificó es que la calidad de la información aumenta el nivel de apoyo. Finalmente, el funcionamiento de la interfaz fue una de los conceptos que más mencionaron los sujetos de estudio.

Con respecto al instrumento creado por Blanchard (2007) es importante mencionar que al comparar los conceptos medidos sólo coinciden las cuatro dimensiones de McMillan y Chavis (1986), la identificación y el apoyo emocional entre miembros. De esta última, no fueron de importancia para los encuestados, la participación de los miembros de la comunidad a través del email, seguramente porque en las actuales comuni- 
dades la mayoría de las participaciones son anónimas y además como lo mencionaron los entrevistados, la relación dentro de la comunidad es más importante, y finalmente el concepto "observación sobre cómo otros comparten información" tampoco fue de relevancia, debido a que el nivel de apoyo depende principalmente de la calidad de la información compartida.

Es interesante ver como algunos conceptos dentro de las anteriores categorías fueron identificados como prioritarios para los encuestados, por ejemplo, el 97\% consideró el entendimiento como un concepto importante, el cual se refiere a la oportunidad de aprender, obtener hablidades y experiencias, el 92\% escogieron el conocimiento, definido como la oportunidad de encontrar información útil en estas $\mathrm{CV}, 89 \%$ comentaron que la carrera era importante (la manera para obtener un mejor desarrollo profesional y una mayor cultura general) y el $84 \%$ mencionó la autoexpresión, como un medio para expresar emociones y ofrecer opiniones. Se sugiere estudiar dichos conceptos con mayor profundidad en investigaciones futuras para confirmar si influyen en el sentido de comunidad virtual.

Definitivamente el resultado de esta investigación permitirá crear una escala más confiable, que nos permita monitorear el sentido de comunidad virtual de la tal manera que se puedan hacer ajustes y se logre mantenerlas para preservar la información compartida y la generación del conocimiento en beneficio de la sociedad.

\section{REFERENCIAS}

Bingshan, Y. (2010). Research on influencing factors of members sense of community in virtual professional communities. International Journal of Continuing Education and Lifelong Learning, 2(2), 101-116.

Blanchard, A., \& Markus, M. (2002). Sense of virtual community-maintaining the experience of belonging. Investigación presentada en el Proceedings of the Annual Hawaii International Conference on System Sciences, 270. Recuperado el 30 de mayo de 2009 de la base de datos British Library Document Supply Centre Inside Serials \& Conference Proceedings.

Blanchard, A. L. \& Markus, M.L. (2004, Winter). The experienced "sense" of a virtual community: Characteristics and processes. ACM SIGMIS Database, 35(1), 65-79. Recuperado el 13 de abril de 2009 de las base de datos ACM.DIGITAL LIBRARY.

Blanchard, A.L. (2007). Developing a sense of virtual community measure. Ciberpsychology \& Behavior, $10(6), 827-830$.

Blanchard, A. L. (2008). Testing a model of sense of virtual community. Computers in Human Behavior, 24 (5), 2107-2123. Recuperado el 26 de octubre de 2008 de la base de datos Psycinfo.

Brunold, J., Merz, H. \& Wagner, J. (2002). Comunidades virtuales: Parte fundamental de la estrategia del negocio electrónico. España: Ediciones Deusto.
Chavis, D. M., Hogge, J. H., McMillan, D. W. \& Wandersman, A. (1986, enero). Sense of community through Brunswik's lens: A first look. Journal of Community Psychology, 14 (1), 24-40.

Chavis, D. M. \& Newbrough, J. R. (1986). The meaning of "community" in community psychology. Journal of Community Psychology, 14 (4), 335-340. Recuperado el 20 de junio de 2009 de la base de datos SOCIndex.

Cheon, E. \& Ahn, J. (2009). Virtual community 101: Know your virtual community and members. Conferencia presentada en el ICUIMC-09. Enero 15-16. Recuperado el 20 de junio de 2010 de la base de datos ACM.

Chin, A. \& Chignell, M. (2007, junio). Identifying communities in blogs: roles for social network analysis and survey instruments. International Journal of Web Based Communities, 3 (3), 345-363. Recuperado el 28 de octubre de 2008, de la base de datos Wiley Interscience.

Community. (2010). In Merriam-Webster Online Dictionary. Recuperado el 12 de abril de 2010 de http:// www.merriam-webster.com/dictionary/community

Ellonen. H., Kosonen \& Henttonen (2007). The development of a sense of virtual community. International Journal of Web Based Communities, 3 (1), 114-130. Recuperado el 10 de mayo de 2009 de las base de datos ACM. 
Fisher, A. T., Sonn, Ch. C. \& Bishop, B.J. (2002). Psychological sense of community: Research, applications, and implications. New York: Kluwer Academic/Plenum Publishers.

Garber, D. (2004, agosto). Growing virtual communities. International Review of Research in Open and Distance Learning, 7. Recuperado el 23 de marzo de 2008, de http://www.irrodl.org/content/v5.2/technote4.html.

Glynn, T.J. (1981). Psychological sense of community: Measurement and application. Human Relations, 34, 780-818.

Koh, J.; Kim, Y. G. (2003). Sense of virtual community: A conceptual framework and empirical validation. International. Journal of Electronic Commerce, 8 (2), 75-93.

Koh, J.; Kim, Y. G, Butler, B. \& Bock, G-W. (2007, febrero). Encouraging participation in virtual communities. Communication of the ACM, 50 (2), 69-73.

Kruger, L. J., Maital, S. L., Macklem, G., Shriberg, D., Burgess, D. M., Kalinsky, R. \& Corcoran, K. (2001). Sense of community among school psychologists on an Internet site. Professional Psychology: Research and Practice, 32 (6), 642-649.

Moore, T. D., \& Serva, M. A. (2007). Understanding member motivation for contributing to different types of virtual communities: A proposed framework. Investigación presentada en SIGMIS-CPR'07, April 19-21, (pp. 153-158). St. Louis, Missouri, USA.

Morales, D. (2010, 11 de octubre). Atrae más Internet que televisión a jóvenes. Cuarto Poder Diario de Chiapas. Recuperado el 13 de abril de 2011 de la base de datos de In 4 mex.

Nielsen, J. (2006, octubre). Participation inequality: encouraging more users to contribute. Recuperado el 20 de junio de 2009 de: http://www.useit.com/alertbox/ participation_inequality.html

Obst, P. (2002). Community connections: psychological sense of community and identification in geographical and relational settings. Tesis de doctorado. School of Psychology and Counselling. Queensland University of Technology. Australia. Recuperado el 24 de marzo de 2008, de http://adt.library.qut.edu.au/adt-qut/ public/adt-QUT20050615.113000/

Obst, P. L. \& White, K. M. (2004). Revisiting the sense of community index: A confirmatory factor analysis.
Journal of Community Psychology, 32 (6), 691-705. Recuperado el 6 de octubre de 2008 de la base de datos Wiley Interscience.

O'Reilly, T. (2005). What is web 2.0. Recuperado el 20 de junio de 2009, de http://oreilly.com/web2/archive/ what-is-web-20.html

Porter, C.E. (2004, noviembre). A typology of virtual communities: A multi-disciplinary foundation for future research. JCMC, 10 (1). Recuperado el 2 de abril de 2008, de http://jcmc.indiana.edu/vol10/issue1/porter.html\#introduction

Rheingold, H. (1993). A slice of life in my virtual community. En L. M. Harasim (Ed.). Global Networks: Computer and International Communication. Cambridge, MA. The MIT Press.

Rheingold, H. (2006). La comunidad virtual. España: Edisa.

Rheingold, H. (2010). Howard Rheingold. Recuperado el 10 de junio de 2010 de http://www.rheingold.com/

Shauhnessy, J., Zechmeister, E. \& Zechmeister, J. (2007). Métodos de investigación en psicología. México: McGraw-Hill.

Tilley, Ch. M. (2006). A sense of control: A model of virtual community for people with mobility impairments. Tesis de doctorado. School of Information Systems. Queensland University of Technology. Australia. Recuperado el 24 de marzo de 2008, de http://adt. library.qut.edu.au/adt-qut/public/adt-QUT200705 $15.155616 /$

Wang, J. Ch. \& Chen, Ch. L. (2004). An automated tool for managing interactions in virtual communities, using social network: Analysis aproach. Journal of Organization Computing and Electronic Commerce, $14(1), 1-26$.

Yu, J., Jiang, Z. \& Chan, H. Ch. (2007, 19-21 de abril). Knowledge contribution in problem solving virtual communities: The mediating role of individual motivation. Investigación presentada en SIGMIS.

Fecha de recepción: Diciembre 2010 Fecha de publicación: Abril 2011 